This is the accepted manuscript of the following article: Freire, S., de Araujo, M., Al-Soufi, W., \& Novo, M. (2014). Photophysical study of Thioflavin T as fluorescence marker of amyloid fibrils. Dyes And Pigments, 110, 97-105. doi: 10.1016/j.dyepig.2014.05.004. (c) 2014 Elsevier B.V.

This manuscript version is made available under the CC-BY-NC-ND 4.0 license (http://creativecommons.org/licenses/by-nc$\mathrm{nd} / 4.0 /)$

\title{
Photophysical study of Thioflavin $T$ as fluorescence marker of amyloid fibrils
}

Sonia Freire, Marcus H. de Araujo, Wajih Al-Soufi and Mercedes Novo*

Departamento de Química Física, Facultade de Ciencias, Universidade de Santiago de

Compostela, E-27002 Lugo (Spain), Tel. (+34) 982824084, Fax: (+34) 982824001,

E-mail: m.novo@usc.es

\begin{abstract}
Thioflavin $\mathrm{T}$ is a highly sensitive fluorescent marker of amyloid fibrils that has been widely used for in vitro biomedical assays. However, neither its complex photophysical behavior nor its binding mode to amyloid fibrils are still well understood. We present a detailed analysis of the photophysical properties of Thioflavin $\mathrm{T}$ in various media, including solvents and solvent mixtures of different viscosities as well as fibrillar and globular proteins. We propose a model that explains the strong wavelength dependency of the Thioflavin $\mathrm{T}$ fluorescence and the large fluorescence enhancement in certain environments. We determine the binding affinities and the fluorescence properties of Thioflavin T bound to amyloid- $\beta$ (1-42) fibrils and to bovine serum albumin and discuss the sensitivity and the specificity of this probe to amyloid aggregates. These results allow us to assess the suitability of Thioflavin $\mathrm{T}$ for quantitative determinations in biomedical studies.
\end{abstract}

\section{Keywords}

Thioflavin T, amyloid fibril, fluorescent marker, photophysical properties, molecular rotor

\section{Abbreviations}

A $\beta 40$ - Amyloid- $\beta$ (1-40), with 40 aminoacids

A $\beta 42$ - Amyloid- $\beta$ (1-42), with 42 aminoacids

BSA - Bovine serum albumin

Buffer sol. - Buffer solution (PBS buffer of pH 7.2)

CT - Charge transfer 
DMSO - Dimethyl sulfoxide

GA - Global Analysis

HAS - Human serum albumin

HFIP - Hexafluoroisopropanol

LE - Locally excited state

PBS - Phosphate buffered saline

PCA - Principal Components Analysis

PCGA - Principal Components Global Analysis

ThT - Thioflavin T

TICT - Twisted intramolecular charge-transfer 


\section{Introduction}

The term amyloidosis refers to a group of diseases caused by misfolded proteins that accumulate in various tissues and organs leading to disruption of their normal function. These proteins form insoluble aggregates, called amyloid fibrils, which are characterized by a fibrillar morphology, $\beta$-sheet-rich structure and green birefringence upon staining with Congo Red [1]. The most common marker used for the detection of amyloid fibrils is Thioflavin T (ThT), a fluorescent dye of the benzothiazole family that shows a huge fluorescence enhancement upon binding to amyloid aggregates [2,3]. This dye can be used both for visualization and quantification of amyloid fibrils in vitro using fluorescence spectroscopy and for amyloid diagnosis in tissue sections using fluorescence microscopy [2]. ThT has also been used to monitor the fibrillation process [4,5], although recent studies suggest that the dye itself may promote amyloid aggregation [6]. Another disadvantage of ThT is its lack of interaction with amyloid oligomers and protofibrils [7], which have been proposed to be responsible for neurotoxicity rather than fibrils [8-10].

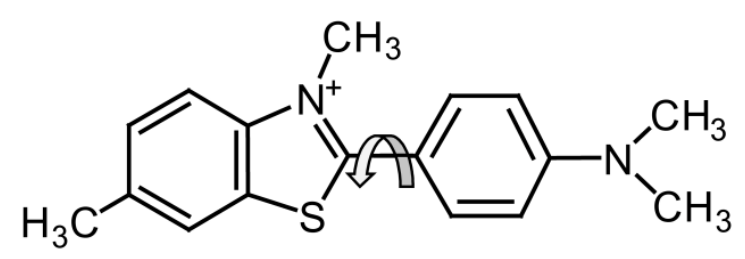

Scheme 1. Molecular structure of Thioflavin $\mathrm{T}$ with the torsion angle $\varphi$ between the benzothiazole and the aminobenzene moieties.

The photophysical mechanism underlying the dramatic fluorescence enhancement of ThT upon binding to amyloid fibrils has been widely studied but is still controversial. Numerous studies postulate that ThT behaves as a molecular rotor, so that its fluorescence properties are determined by the extent of quenching of the locally excited (LE) state to a dark twisted intramolecular charge-transfer (TICT) state [3]. This quenching implies the twisting around the central $\mathrm{C}-\mathrm{C}$ 
single bond (torsion angle $\varphi$ ) in the excited state [11], and is therefore highly dependent on the viscosity of the medium. The observed fluorescence enhancement of ThT in viscous solvents and in different confined environments, where the twisting process is hindered, support this hypothesis, as well as the low fluorescence quantum yields of this dye in non-viscous solvents [12-19]. Also the excited-state dynamics from LE to TICT state have been recently found to be correlated with the viscosity of the medium [20].

Notwithstanding this well founded hypothesis, in the last few years some reports have attributed the changes of ThT fluorescence upon binding to amyloid fibrils to the formation of highly fluorescent dimers, excimers or even micelles of ThT molecules [2,21]. Excimer formation is very unlikely since the lifetime of ThT bound to amyloid fibrils is too short. Also the formation of micelles by ThT is very doubtful and is not clearly supported by the published conductivity data [22]. Hence, in our opinion, the only plausible alternative hypothesis proposed so far would be that ThT binds to amyloid fibrils as dimers. However, dimers of ThT have still not been experimentally detected [23].

The binding mode of ThT to amyloid fibrils has been extensively studied in order to achieve a better structural understanding of amyloid fibrils as well as to explore the potential therapeutic use of this dye. Nevertheless, to date there is no conclusive model that accounts for the orientation and conformation of ThT when bound to amyloid fibrils [2]. Confocal microscopy studies suggested that ThT binds as monomer to amyloid fibrils such that their long axes are parallel [24]. On the basis of crystal structures contradictory results have been reported [25,26], but they are compatible with the existence of a binding region of ThT at the amyloid fibril surface [21]. Very recently the results obtained by Polarization-Resolved Fluorescence Microscopy using ThT as marker revealed polymorphism of insulin amyloid fibrils [27].

Among the many different amyloid-like peptides, those called amyloid- $\beta$ were given most attention due to their primal role in the development of Alzheimer`s disease as they accumulate as insoluble plaques among the neurons at the brain [28]. These deposits are formed mainly by 
peptides of 40 aminoacids (A $\beta 40)$ and 42 aminoacids (A $\beta 42)$, the latter being at a higher proportion [28-30]. The A $\beta 42$ shows a higher hydrophobicity and a much lower critical concentration for aggregation than $\mathrm{A} \beta 40$, so that it aggregates very fast and has a high tendency to form fibrils [10,31,32]. In spite of its arduous handling, we have used $A \beta 42$ in this work due to its greater relevance in amyloid aggregation.

\section{Material and method}

\subsection{Materials}

Thioflavin T (ThT, Fluka) was used without further purification. Bovine serum albumin (BSA) was purchased from Sigma-Aldrich and human amyloid- $\beta$ (1-42) (A $\beta 42)$ from GenScript USA Inc. Phosphate buffered saline (PBS, $\mathrm{pH}=7.2,150 \mathrm{mM} \mathrm{NaCl}$ ) was used for the preparation of the amyloid- $\beta$ samples. The solvents used, chloroform (Sigma-Aldrich) and glycerol (Panreac), had a purity of 99\%. All aqueous solutions were prepared using Milli-Q water.

\subsection{Sample Preparation}

Stock solutions of ThT in the different solvents or solvent mixtures were prepared by weighing. The real dye concentration was determined experimentally from the absorbance in water using the reported value of $36,000 \mathrm{M}^{-1} \mathrm{~cm}^{-1}$ for the molar extinction coefficient of ThT in water at 412 $\mathrm{nm}$ [2]. These stock solutions were used within a few days and diluted to the adequate concentration in each experiment. The measurements showed in this work were performed at the following ThT concentrations: (a) PBS samples: 1.2 and $9.4 \mu \mathrm{M}$; (b) chloroform samples: 1-2 $\mu \mathrm{M}$; (c) Glycerol-water mixtures: $2.2 \mu \mathrm{M}$; (d) samples with $\mathrm{A} \beta 42$ fibrils: 1.1 and $7.0 \mu \mathrm{M}$ for fluorescence and $244 \mu \mathrm{M}$ for absorption measurements; (e) samples with BSA: 2.4 and $3.1 \mu \mathrm{M}$ for fluorescence and 12.2 $\mu \mathrm{M}$ for absorption and lifetime measurements.

Stock solutions of BSA and fibrillar A $\beta 42$ were freshly prepared for each experiment. The samples for the titrations were prepared by the dilution-extraction method beginning with the corresponding protein stock solution containing the desired concentration of ThT and diluting 
with a solution of ThT of the same concentration. Amyloid concentrations mentioned in this work refer to the total concentration of amyloid monomers used in the preparation of the sample.

For the preparation of amyloid- $\beta$ fibrils we followed established protocols [33]. Firstly, potential aggregates that could be formed in the commercial peptide were broken up by hexafluoroisopropanol (HFIP). The amyloid was dissolved in HFIP at a concentration of $1 \mathrm{mg} / \mathrm{ml}$ and the solution was incubated during 1 hour with occasional mixing and then shaken for about 20 minutes. Next the solution was split up into several vials and the HFIP was removed under a stream of nitrogen. Vials were transferred into a desiccator and vacuum was applied to remove the remaining traces of HFIP. The resulting dried aliquots of monomeric peptide were stored at $20^{\circ} \mathrm{C}$.

Stock solutions of the amyloid were prepared by suspending the peptide aliquot in dried dimethyl sulfoxide (DMSO) with a final concentration of $\approx 1 \mathrm{mM}$ and shaking it for about 15 minutes in order to dissolve the peptide. To prepare $\beta$-amyloid fibrils an aliquot of the DMSO stock solution was dissolved in PBS at $\mathrm{pH}$ 7.2. This sample was incubated at $37{ }^{\circ} \mathrm{C}$ for 2 days under vigorous agitation.

\subsection{Absorption and Fluorescence Measurements}

Absorption spectra were recorded using quartz cells with path lengths of 3.00 and $10.0 \mathrm{~mm}$ in a Varian-Cary 100 spectrometer. All spectra were recorded using baseline correction with the corresponding solvent. Nevertheless, for the titrations with BSA and A $\beta 42$, further correction of the experimental absorption spectra was required in order to subtract the contributions of protein absorption and light dispersion (only for the samples with A $\beta 42$ ). The absorption spectra are given in units of molar absorptivity.

Steady-state and time-resolved fluorescence measurements were performed using an EdinburghInstruments F900 spectrofluorimeter, equipped with a Xenon lamp of $450 \mathrm{~W}$ as excitation source for steady-state measurements and a picosecond diode laser 405-nm laser (Becker \& Hickl 
$\mathrm{GmbH}, \approx 80 \mathrm{ps}, 20 \mathrm{MHz}$ ) for lifetime measurements with the time-correlated single photon counting technique. Excitation and emission fluorescence spectra were corrected for the wavelength-dependent output of the Xenon lamp and the non-ideal response of the instrument, respectively. Additionally, all spectra were corrected for the solvent contribution, in order to remove the intense Raman bands appearing in the samples of very low quantum yields and the fluorescence of impurities in the samples with glycerol. The contribution of BSA and A $\beta 42$ autofluorescence was also subtracted. Fluorescence quantum yields were determined using Quinine Sulphate ( $\phi=0.58$ in $0.1 \mathrm{M}$ sulfuric acid) [34] or Coumarin $152(\phi=0.064)$ [35] as standards for excitation wavelengths below and above $400 \mathrm{~nm}$, respectively. For anisotropy measurements Glan-Thompson polarizers were used with a spectral range of 230-900 nm.

Both absorption and fluorescence measurements were carried out at $25 \pm 1{ }^{\circ} \mathrm{C}$.

\subsection{Data analysis}

Most data processing was done with the program OriginPro 8.5 (OriginLab Corporation, US). The series of absorption and emission spectra obtained in the titrations were analyzed using a program developed in our group which applies Principal Components Global Analysis (PCGA) [36]. The first step of PCGA is the Principal Components Analysis (PCA) which yields the minimal number of components that reproduce the experimental spectra, i.e. it allows one to determine the number of chemical species that contribute to the spectra. This step helps to propose a mechanism which explains the observed variations. Global Analysis (GA) consists in the fit of the proposed theoretical model to the whole series of experimental spectra in order to estimate the model parameters and the pure spectra of the contributing species.

Individual fits of fluorescence decays were performed with the software package from Edinburgh Instruments. The series of fluorescence decays measured at different protein concentrations together with the corresponding pulse responses were also fitted globally using a home-made program in Matlab (The MathWorks, US). 
The values of refractive index and viscosity of the solvents and glycerol-water mixtures were obtained from the literature [37-39].

Different properties $Y$ (absorbance, fluorescence intensity, ...) were related to protein concentration $[\mathrm{H}]$ by a 1:1 complexation model with binding equilibrium constant $K$ and the limiting values of the property for free and bound dye, $A$ and $B$, respectively:

$$
Y=\frac{A+B \cdot K \cdot[\mathrm{H}]}{1+K[\mathrm{H}]}
$$

\section{Results and discussion}

The photophysical behavior of ThT is very complex, already in aqueous solution, but even more in the presence of BSA or A $\beta 42$-fibrils. In order to understand it the full picture has to be analyzed, comparing and explaining critically the results from multiple techniques in different solvents and environments. In the following the absorption, steady-state fluorescence emission, excitation and anisotropy spectra are analyzed in different media as function of excitation and emission wavelength, concentrations of BSA and A $\beta 42$-fibrils as hosts and the solvent viscosity in glycerol water mixtures. A model is described which gives a good explanation of the experimental findings and which is in line with results published by other authors.

\subsection{Wavelength dependency of ThT fluorescence and absorption}

Figure 1 shows fluorescence emission spectra measured at different excitation wavelengths, together with excitation spectra monitored at two emission wavelengths and the absorption spectra, of ThT in three environments: aqueous solution, chloroform and a concentrated solution of amyloid fibrils. Chloroform was chosen as an aprotic and relatively nonpolar solvent, opposite to water. Fluorescence emission maxima, quantum yields and anisotropy determined for each excitation wavelength are given in Table 1. 

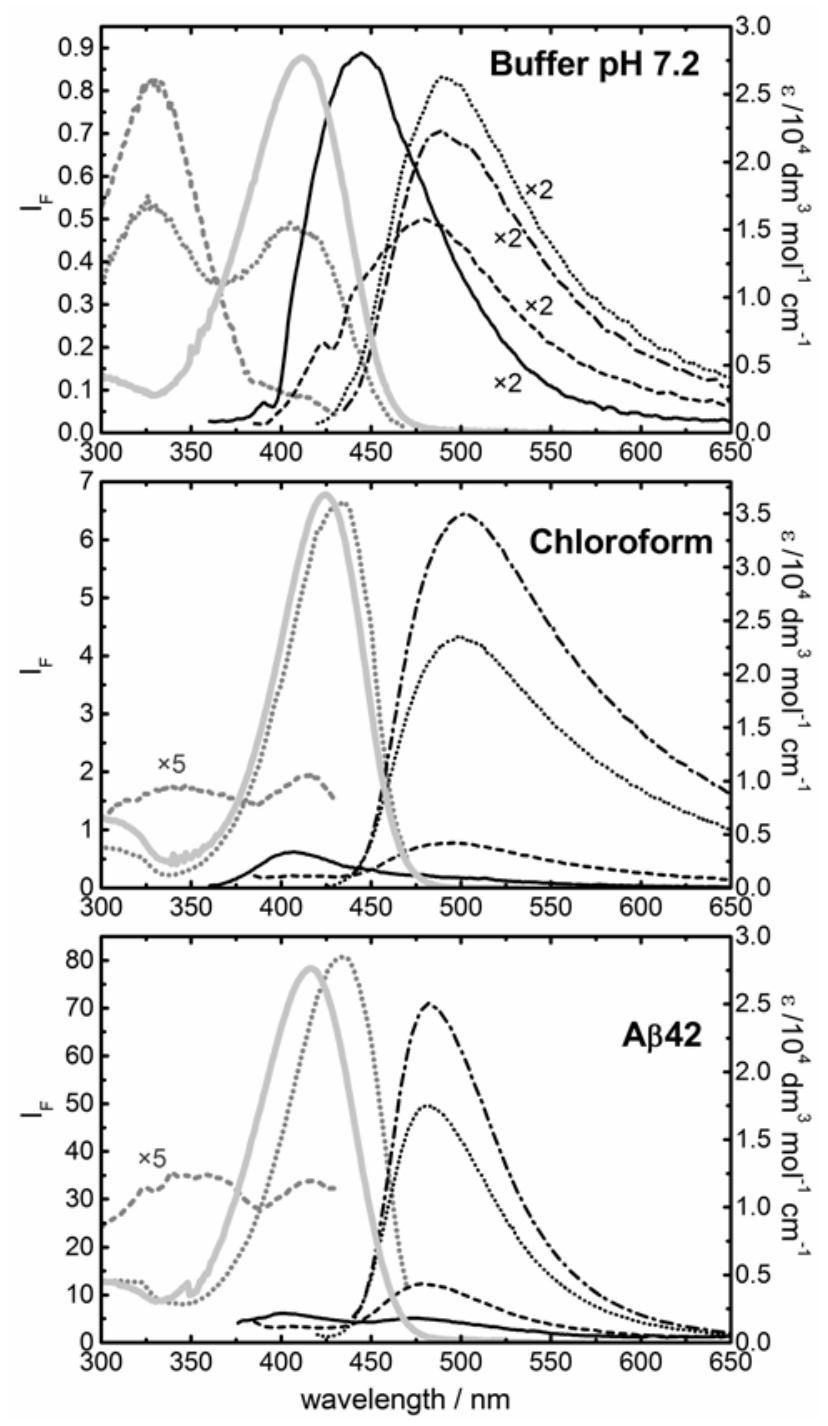

Figure 1. Absorption (light gray solid lines) and fluorescence excitation (dark gray lines on the left) and emission (black lines on the right) spectra of ThT in PBS buffer, chloroform and in the presence of an aqueous solution of A $\beta 42138 \mu \mathrm{M}$. Excitation spectra monitored at 440-nm (grey dashed lines) and 480-nm (grey dotted lines). Emission spectra measured with excitation at $350 \mathrm{~nm}$ (black solid lines), $375 \mathrm{~nm}$ (black dashed lines), $410 \mathrm{~nm}$ (black dotted lines) and $430 \mathrm{~nm}$ (black dash-dot-dashed lines). Fluorescence intensity (left scale) was measured under the same experimental conditions for all three samples. Absorption spectra are given in units of molar absorptivity (right scale). Some spectra were multiplied by factors as indicated. 
Table 1. Photophysical properties of ThT in aqueous solution (PBS buffer of $\mathrm{pH}$ 7.2), chloroform and a solution of $\mathrm{A} \beta 42138 \mu \mathrm{M}$ : absorption maximum $\lambda_{a b}^{\max }$, emission maxima for different excitation wavelengths $\lambda_{e m}^{\max }$, fluorescence quantum yield $\phi$, and steady-state anisotropy $r$ in the wavelength intervals $425-450 \mathrm{~nm}$ and $500-525 \mathrm{~nm}$. The anisotropy values in brackets are averages of data with a clear tendency.

\begin{tabular}{|c|c|c|c|c|c|c|}
\hline Thioflavin $\mathrm{T}$ & $\lambda_{a b}^{\max } / \mathrm{nm}$ & $\lambda_{e x} / \mathrm{nm}$ & $\lambda_{e m}^{\max } / \mathrm{nm}$ & $\phi$ & $r(425-450 \mathrm{~nm})$ & $r(500-525 \mathrm{~nm})$ \\
\hline \multirow{3}{*}{ Buffer solution } & \multirow{3}{*}{412} & 350 & 445 & 0.0017 & $\approx 0$ & $(0.21)$ \\
\hline & & 375 & 479 & 0.00086 & - & $0.31 \pm 0.02$ \\
\hline & & 410 & 489 & 0.00033 & - & $0.36 \pm 0.01$ \\
\hline \multirow{3}{*}{ Chloroform } & \multirow{3}{*}{425} & 350 & 407 & 0.016 & $0.09 \pm 0.01$ & $(0.15)$ \\
\hline & & 375 & 491 & 0.013 & - & $0.167 \pm 0.006$ \\
\hline & & 410 & 498 & 0.017 & - & $0.149 \pm 0.005$ \\
\hline \multirow{3}{*}{$\begin{array}{l}\text { Fibrillar } \\
\beta \text {-amyloid(1-42) } \\
{[\mathrm{A} \beta 42]=138 \mu \mathrm{M}}\end{array}$} & \multirow{3}{*}{416} & 350 & $\begin{array}{l}402 \\
472\end{array}$ & 0.077 & $0.252 \pm 0.007$ & $(0.31)$ \\
\hline & & 375 & 479 & 0.035 & - & $0.347 \pm 0.006$ \\
\hline & & 410 & 482 & 0.048 & - & $0.350 \pm 0.002$ \\
\hline
\end{tabular}

The absorption spectra of ThT are very similar in the three samples, with only small shifts of their maxima $\lambda_{a b}^{\max }$. The emission spectrum of ThT depends in all three media strongly on the excitation wavelength (black curves in Figure 1). When exciting at $350 \mathrm{~nm}$, in the minimum of the absorption spectrum, an emission band in the range of $400-450 \mathrm{~nm}$ is observed (black solid curve), whereas excitation at the high absorbance wavelengths longer than $400 \mathrm{~nm}$, leads to an emission band around 480-500 nm (black dotted and dash-dotted curves). These two emission bands are observed as a mixture when exciting in-between, for example at $375 \mathrm{~nm}$ (black dashed curve). In accordance to this, the observed emission maxima of ThT depend on the excitation wavelength in all three media ( $\lambda_{e m}^{\max }$ in Table 1$)$. Moreover, the fluorescence quantum yields determined from these experimental emission spectra show significant differences depending on the excitation conditions ( $\phi$ in Table 1). It is remarkable that the fluorescence quantum yield at 350-nm excitation is comparably high, in spite of the low or similar intensity of the corresponding 
emission spectra. This turns out to be the result of the much lower absorbance of ThT at 350nm as compared to the other excitation wavelengths (see solid grey curve in Figure 1).

So far, the dependency of the emission spectra on the excitation wavelength hints to the presence of at least two ground-state species of ThT of different photophysical properties, one (type 1) with low absorbance at lower wavelengths but a comparably high quantum yield and another (type 2) with high absorbance at higher wavelengths but lower quantum yield.

This observation is confirmed by the excitation spectra. In PBS buffer the excitation spectra have always a well-defined band at $330 \mathrm{~nm}$ that decreases in intensity as the recording emission wavelength is increased, at the same time that a second excitation band at about $410 \mathrm{~nm}$ appears. The excitation spectrum monitored at $440 \mathrm{~nm}$ (grey dashed curve) would be due to the first, highly emissive species, absorbing very weakly in the $300-350 \mathrm{~nm}$ range, which is therefore not detected in the absorption spectra. The excitation band followed in the 480-nm emission band (grey dotted curve) corresponds then to a mixture of the emissions of the first species and of the second, strongly absorbing species that is the main responsible for the overall observed absorption.

For chloroform and amyloid fibrils the behavior is similar, although the short-wavelength excitation and emission bands are less intense than in aqueous solution and two emission bands can be distinguished when exciting at $350 \mathrm{~nm}$ (black solid curves in Figure 1). In buffer, the much higher fluorescence quantum yield of the first species, emitting at short wavelengths, in comparison to the second, red emitting species (Table 1) explains then that the short-wavelength excitation band is so visible in aqueous solution.

A first, simple hypothesis that assigned the short-wavelength excitation and emission bands to impurities [40] is not convincing since these bands are still present with similar contributions after thorough recrystallization of ThT [2].

Recently, Maskevich et al. proposed an alternative explanation for the wavelength dependency of ThT fluorescence in aqueous and alcoholic solutions, which is based on the existence of two 
types of ground-state conformers: one with a torsion angle $\varphi$ between the benzothiazole and the aminobenzene moieties (Scheme 1) close to $90^{\circ}$ or $270^{\circ}$, and another one with a unified $\pi$ electron system corresponding to the energy minima at the torsion angles of $37^{\circ}$ and $145^{\circ}$, and $217^{\circ}$ and $325^{\circ}$ [12]. In the first type of conformers the benzothiazole and aminobenzene rings would behave as independent chromophores and therefore, show absorption and emission bands at shorter wavelengths than the second type of conformers with an integrated system of $\pi$ conjugated bonds, which would be responsible for the observed strong absorption band and the corresponding long-wavelength, low-energy, emission. Quantum-chemical calculations showed that the methyl group bound to the nitrogen of the benzothiazole ring accounts for the low energy barrier separating the two types of conformers with minimum energy, so that the fraction of conformers with torsion angle about $90^{\circ}$ or $270^{\circ}$ is not negligible at room temperature [41]. These are, however, results obtained for the gas phase.

The two species observed in the emission and excitation spectra can be readily assigned to the two conformers. This model explains very nicely the basic photophysical behavior of ThT observed so far in the different environments.

However, some experimental details show that this model with only two, well defined, conformers is not sufficient. The excitation spectra measured in chloroform and with $A \beta 42$ at the longer 480-nm emission are atypical, as far as they show a small but significant red shift with respect to the corresponding absorption spectrum. For amyloid solutions it was proven that this red shift increases further with increasing monitoring emission wavelength (data not shown). In a two species model the absorption spectrum would be the weighted mean of that of the two species and would be expected to be broader but not shifted with respect to the excitation/absorption spectrum of one of the species.

The influence of the solvent polarity on the absorption and emission maxima is small, except for the emission at short wavelengths that is blue shifted in chloroform and amyloid fibrils as 
compared to aqueous solution (Table 1). On the contrary, a great effect is observed in the fluorescence quantum yields that are at least one order of magnitude higher in chloroform than in buffer.

Anisotropy emission spectra determined for the different excitation wavelengths give additional information about the photophysical behavior of ThT in the different media. In general, the anisotropy of the ThT fluorescence does not have a constant value throughout the emission range (Figure 1S in SI). When exciting at $350 \mathrm{~nm}$ the mean anisotropy of the short-wavelength emission can be determined in the range from 425 to $450 \mathrm{~nm}$ (Table 1), whereas increasing values are obtained at longer wavelengths. Excitation at $375 \mathrm{~nm}$ and $410 \mathrm{~nm}$ yields constant values of the anisotropy for the emission at long wavelengths (anisotropy in the range 500-525 nm, Table 1), which are higher than those of the short-wavelength emission. Both in aqueous solution and in the presence of amyloid the mean anisotropies at long wavelengths coincide and approach the anisotropy limit, whereas in chloroform they are significantly lower.

On the basis of these observations, an extension of the model described above is proposed (Scheme 2). Instead of two conformers, ThT presents at room temperature in a given environment a wider distribution of ground-state conformations, going from those with nearly perpendicular benzothiazole and aminobenzene moieties $\left(\varphi=90^{\circ}\right.$ or $\left.270^{\circ}\right)$, which are responsible for excitation/emission at short wavelengths, to those almost planar $\left(\varphi=0^{\circ}\right.$ or $\left.180^{\circ}\right)$, which correspond to excitation/emission at long wavelengths. The distribution of molecules among the different possible conformations depends in homogeneous solutions on the solvent polarity and temperature, being the perpendicular ones better stabilized in polar solvents. In heterogeneous solutions (BSA, A 442 ) the distribution depends also on the local microenvironment of ThT. 


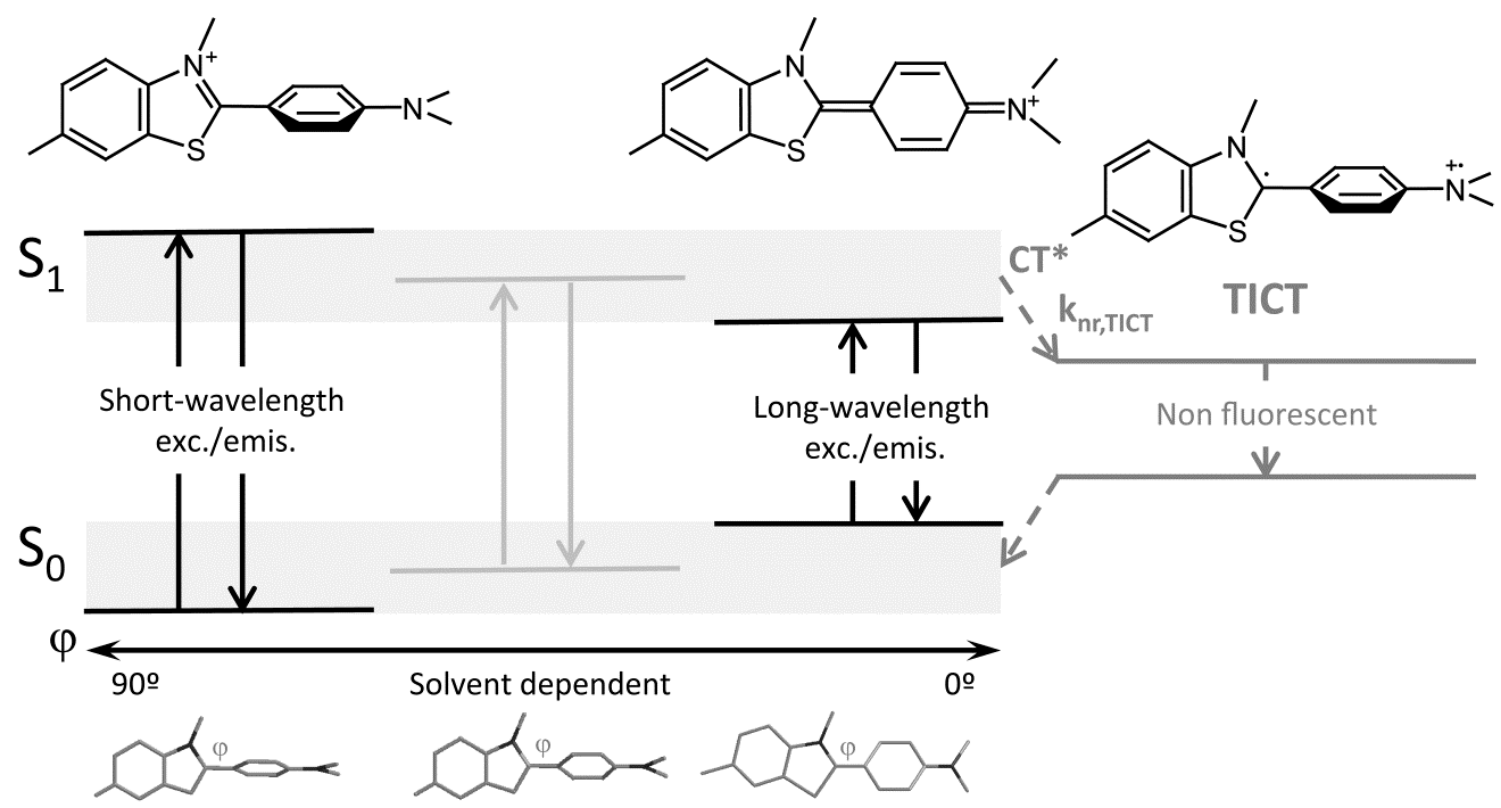

Scheme 2. Model proposed to explain the ground and excited-state behavior of ThT, which presents a distribution of conformations with different photophysical properties. Planar conformations can undergo charge transfer (CT) in the excited state to the nonfluorescent TICT state.

The observed absorption spectra are linear combinations of the spectra of the different conformers and depend therefore exclusively on their equilibrium concentrations and their molar absorptivities. The positions of the absorption maxima (Table 1) show that, in all three media, conformations approaching planarity that allow $\pi$-conjugation predominate in the ground state and are moderately dependent on the solvent polarity, as stated in the literature [12].

The strong wavelength dependency of the excitation and emission spectra is due to the very different excited-state behavior of the conformers that, in turn, depends strongly on solvent polarity and viscosity. Depending on the excitation wavelength, different conformational subpopulations are selected. At shorter wavelength ThT molecules with rather perpendicular conformations are excited, which show the typical emission of the benzothiazole moiety and its corresponding excitation band, characterized by relatively high fluorescence quantum yield and 
strong dependency on solvent polarity [42]. These conformations are favored in aqueous solution in comparison to chloroform, and are also observed in the concentrated amyloid solution. The blue-shifted emission maximum, higher fluorescence quantum yield and increased anisotropy of ThT in the A $\beta 42$ solution with respect to the buffer solution (Figure 1 and Table 1 ) indicate that such conformers bind to the amyloid fibrils.

ThT molecules excited at longer wavelengths have close to planar conformations and emit from the locally excited state or deactivate by charge transfer to the nonfluorescent TICT state. Studies with similar benzothiazole derivatives support this mechanism and show that the presence of the methyl group at the nitrogen of the benzothiazole increases the electron-acceptor strength of this moiety as well as the amino group of the benzene moiety increases its electron-donor capacity $[43,44]$. The extent of the charge-transfer quenching process depends on the polarity of the medium, but, above all, on its viscosity or its ability to hinder the excited-state twisting process. In non-viscous solvents, such as water and chloroform, twisting is free and the fluorescence intensity depends on the stabilization of the TICT state by the solvent, which is more effective in aqueous solution. This transition of the excited planar ThT to the nonfluorescent TICT state is the reason for the very low fluorescence quantum yield of ThT in aqueous solution (Table 1). By contrast, ThT bound to amyloid fibrils seems to be locked to nearly planar conformations and cannot undergo the twisting process. Therefore it emits from the locally excited state with high fluorescence quantum yield. The high anisotropy value obtained in this case (Table 1), close to the limit and much higher than that in chloroform, confirms this explanation.

The long-wavelength emission of ThT in aqueous solution presents a very high anisotropy in spite of its free rotation. This might be due to the fact that the observed very weak emission corresponds to the small subpopulation of molecules that have planar conformation in the ground state and emit from the same excited-state conformation without being quenched by rotation.

The selective excitation of specific conformers allows for a plausible explanation of the red shift of the ThT excitation spectra with respect to the absorption spectrum in solutions of A $\beta 42$ fibrils. 
The absorption spectrum in this medium corresponds to the wide distribution of conformers of both free and bound ThT, weighted by concentration and absorptivities. Only a small fraction of ThT is in a conformation with torsion angles low enough to lead to absorption at higher wavelengths. However, the excitation spectrum is additionally weighted by the quantum yield, which is especially high for low torsion angles. Therefore, the excitation spectra recorded at higher emission wavelengths selectively represent those highly emissive conformations, not distinguishable in the absorption spectrum due to their low contribution.

From this notably complex photophysical behavior it can already be concluded that ThT is not a suitable probe for the quantitative determination of amyloid fibrils. All fluorescence properties show a strong wavelength dependency caused by a complex ground and excited-state behavior that makes it difficult to establish reference values. Moreover, the large fluorescence enhancement upon binding to amyloid fibrils that makes ThT a remarkable marker is also a challenge since the fluorescence properties of bound ThT cannot be easily related to those of the practically non-fluorescent free dye.

\subsection{Fluorescence properties of bound Thioflavin T}

In this second part of the work we determine the binding constant of ThT to A $\beta 42$ fibrils and the photophysical properties of the bound dye. These results are compared with those obtained for ThT bound to a non fibrillar protein and with the fluorescence properties of this dye in viscous media. The aim is to examine the difference between the fluorescence of ThT bound to amyloid fibrils as compared to other rigid media as well as to analyze the specificity of the dye to such fibrillar structures. Glycerol-water mixtures were used to obtain homogeneous media of increasing viscosities.

Figure 2 shows the fluorescence titrations of ThT in aqueous solution with increasing volume percentage of glycerol, concentration of BSA or concentration of A $\beta 42$. All measurements were performed under similar excitation conditions around the corresponding absorption maxima so 
that mainly the long-wavelength emission is observed (see above). The emission spectra (grey curves, upper wavelength scale) are very similar in all three media, although broader for glycerol and BSA than for amyloid solutions. The emission maxima are slightly blue shifted in the order glycerol, BSA and amyloid. There is also a huge fluorescence enhancement (open circles in Figure 2) that follows a typical complexation profile both for BSA and for A $\beta 42$. This increase in fluorescence is attributed to the binding of ThT to the peptides and a resulting steric hindrance for the dye to undergo the twisting process. By contrast, in glycerol-water mixtures the increase of the fluorescence intensity becomes only noticeable at high volume percentages of glycerol, where the viscosity increases enough to hinder the formation of the dark TICT state.

Absorption titrations (Figure 2S in SI) show slight but systematic variations of the absorption spectrum of ThT in aqueous solution as the volume percentage of glycerol or the concentrations of BSA or A $\beta 42$ are increased. A red shift is observed in all three media, indication for the redistribution of the ground-state equilibrium conformations with increasing contributions of planar conformations as the rotation is more and more hindered by the medium. In the case of BSA and A $\beta 42$ these variations are due to the binding of ThT and suggest again that approximately planar conformations bind preferably to the peptides. For the glycerol-water mixtures the spectral variations are more significant at low volume percentage of glycerol, in contrast to the fluorescence enhancement (Figure 2), indicating that they are induced by polarity changes rather than by viscosity effects. 

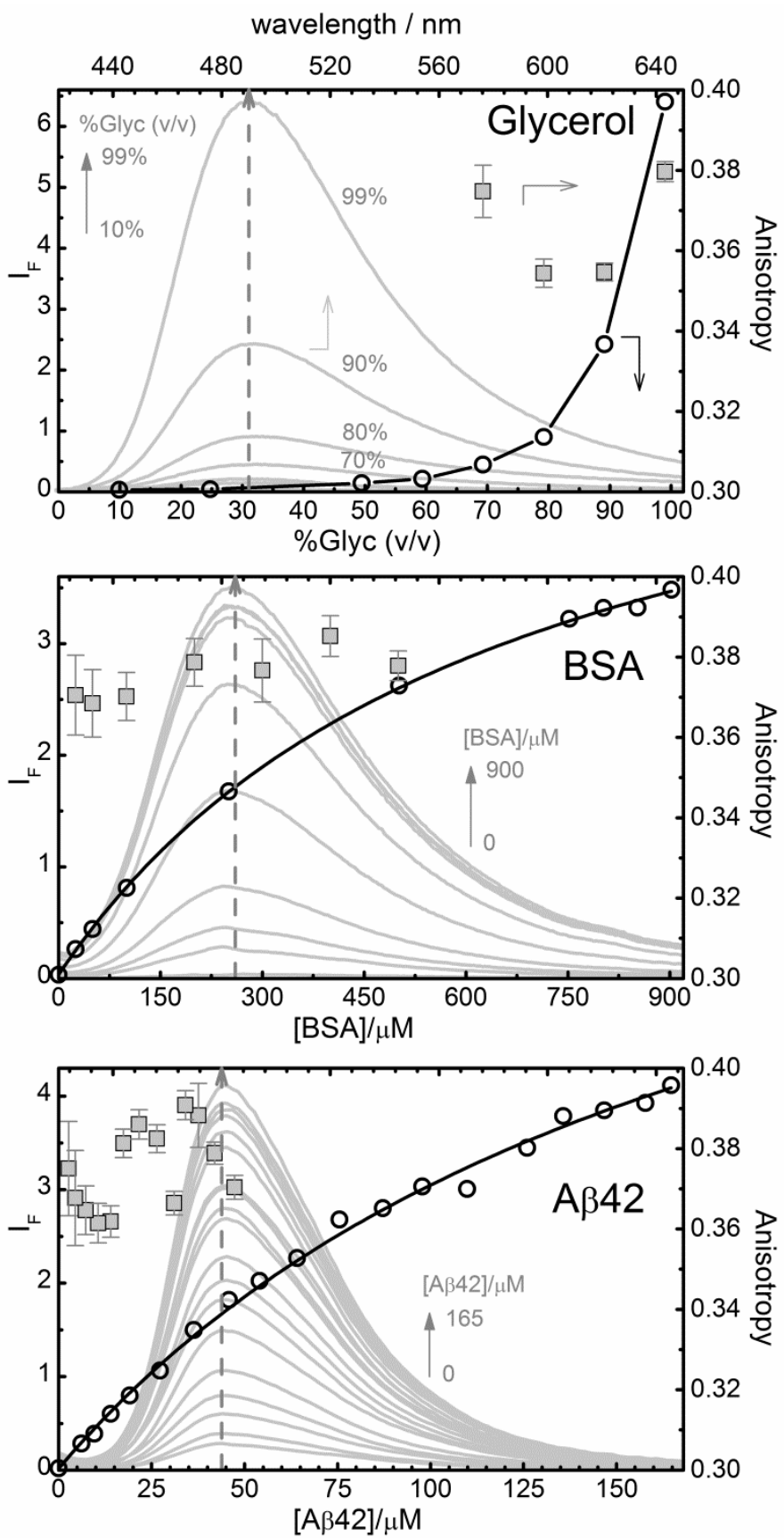

Figure 2. Fluorescence emission spectra (grey lines, upper scale) obtained in the titrations of ThT in aqueous solution with glycerol, BSA and A $\beta 42$. The open circles represent the fluorescence intensities of ThT at the emission maximum as a function of the volume percentage of glycerol or the concentrations of BSA and A $\beta 42$ (lower scale). In the case of BSA and A $\beta 42$ the solid black lines were obtained by the fit of a 1:1 complexation model (eq. (1)) to the experimental data (for 
the parameters see text). The grey squares are the mean anisotropy values $r$ of ThT determined in the emission range 500-525 nm. All measurements were performed with an excitation wavelength of $410 \pm 10 \mathrm{~nm}$, except for the anisotropy at different concentrations of $\mathrm{A} \beta 42$, where $450 \mathrm{~nm}$ was used. Left scale: fluorescence intensity; right scale: anisotropy; bottom scales: volume percent or concentration; top scale: wavelength.

Table 2. Photophysical properties of ThT in different media under excitation at $410 \pm 10 \mathrm{~nm}$ : absorption maximum $\bar{v}_{a b}$, emission maximum $\bar{v}_{e m}$, steady-state anisotropy $r$ (mean values of emission at 500-525 nm), fluorescence quantum yield $\phi$, and fluorescence lifetimes $\tau$ (emission wavelength $=490 \pm 10 \mathrm{~nm}$ ) with their corresponding contribution to fluorescence. The values given for BSA and A $\beta 42$ were obtained from global analysis of the corresponding series of absorption and emission spectra and of fluorescence decays (see text). The last column shows the apparent binding constants $K$ of ThT to BSA and A $\beta 42$.

\begin{tabular}{|c|c|c|c|c|c|c|c|}
\hline ThT & $\bar{v}_{a b} / \mathrm{cm}^{-}$ & $\bar{v}_{e m} / \mathrm{cm}^{-1}$ & $r$ & $\phi$ & $\begin{array}{l}\tau_{1} / \mathrm{ns} \\
\text { (\%fluor) }\end{array}$ & $\begin{array}{l}\tau_{2} / \mathrm{ns} \\
\text { (\%fluor) }\end{array}$ & $\begin{array}{l}\text { K/ } \\
10^{3} \mathrm{~mol}^{-1} \\
\mathrm{dm}^{3}\end{array}$ \\
\hline Buffer sol. & 24270 & 19960 & $0.36 \pm 0.01$ & 0.00033 & $\begin{array}{l}0.023 \pm 0.001 \\
(81)\end{array}$ & $\begin{array}{l}1.42 \pm 0.03 \\
(19)\end{array}$ & - \\
\hline Chloroform & 23530 & 19570 & $0.149 \pm 0.005$ & 0.017 & $\begin{array}{l}0.058 \pm 0.001 \\
(46)\end{array}$ & $\begin{array}{l}0.68 \pm 0.04 \\
(54)\end{array}$ & - \\
\hline 99\% Glycerol & 23530 & 20240 & $0.381 \pm 0.003$ & 0.15 & $\begin{array}{l}0.286 \pm 0.003 \\
(54)\end{array}$ & $\begin{array}{l}0.685 \pm 0.008 \\
(46)\end{array}$ & - \\
\hline BSA & 24100 & 20370 & $0.378 \pm 0.004$ & 0.055 & $\begin{array}{l}0.566 \pm 0.006 \\
(40)\end{array}$ & $\begin{array}{l}2.225 \pm 0.007 \\
(60)\end{array}$ & $1.35 \pm 0.01$ \\
\hline $\begin{array}{l}\text { Fibrillar } \\
\text { A } \beta 42\end{array}$ & 23920 & 20580 & $0.350 \pm 0.002$ & 0.31 & $\begin{array}{l}0.82 \pm 0.01 \\
(10)\end{array}$ & $\begin{array}{l}2.37 \pm 0.01 \\
(90)\end{array}$ & $5.85 \pm 0.07$ \\
\hline
\end{tabular}

In order to obtain the binding equilibrium constants and the spectra of ThT bound to BSA and to A $\beta 42$, the whole series of emission spectra were analyzed with PCGA. PCA confirmed that each series can be reproduced by a linear combination of two components that are assigned to free and bound ThT. Then a 1:1 complexation model (eq. (1)) was used as fit function in the global 
analysis of each series of emission spectra, yielding the binding constant $K$ (Table 2) and the "pure" emission spectra of free and bound dye (Figures 3 and $3 \mathrm{~S}$ in SI). Using a similar procedure, the series of absorption spectra (Figure 2S in SI) were analyzed with PCGA yielding the "pure" absorption spectra also shown in Figure 3 ( $K$ was fixed to the value obtained from emission data since the variations of the absorption spectrum are too small for a free fit of $K$ ). It was checked that the spectra obtained for the free dye coincide with those measured experimentally.

The binding equilibrium constants determined with this analysis are given in Table 2 . They are apparent constants resulting from the contribution of the different microscopic constants of each individual conformation and binding site. In the case of BSA, there is a good agreement with the value found in the literature [16]. No data are available for $A \beta 42$, but the value obtained is significantly lower than those reported for other amyloid fibrils [2]. Nevertheless, it must be noted that most literature values are obtained by linearization methods such as Scatchard plots that imply strong assumptions and have high uncertainties. Moreover the high ThT concentrations that are often used lead to erroneous results, as already pointed out by other authors [12].

On the basis of these binding constants we can discuss the specificity of ThT to amyloid fibrils and asses its cross-sensitivity to other proteins. There are some reports that point to nonspecific binding of ThT but they lack quantitative data [22,24,45]. The affinity of the dye to A $\beta 42$ fibrils is only about 4 times higher than to the $\alpha$-helical protein BSA (Table 2). Furthermore, the binding constant of ThT to human serum albumin (HSA) is about 100 times than to BSA [16]. In a mixture of A $\beta 42$ fibrils with BSA or HSA, a significant fraction of ThT would also bind BSA and almost of the dye would bind HSA. Therefore, the affinity of ThT to amyloid fibrils alone does not support its consideration as a specific probe for such fibrillar structures. We will now analyze the photophysical properties of the dye bound to amyloid fibrils in order to evaluate whether they are distinct enough to specifically discriminate these peptides. 
Figure 3 (and $3 \mathrm{~S}$ in SI) compares the spectra obtained for ThT bound to BSA and to A $\beta 42$ with those measured in other media. Their corresponding maxima are given in Table 2. The absorption spectra (grey curves in Fig. 3) are very similar for all media, with a slight shift of about $700 \mathrm{~cm}^{-1}$ in glycerol and chloroform with respect to aqueous solution and nearly no shift for bound ThT. Thus no strong solvent dependency of the absorption maximum is observed, as stated in the literature [12]. The normalized emission spectra show also only small changes of their maximum with the environment, but are clearly different in their width. ThT bound to A $\beta 42$ (solid black line) has the narrowest fluorescence band of all them, with a half width of $2800 \mathrm{~cm}^{-1}$, significantly smaller than for BSA and glycerol (dotted and dash-dot-dotted lines, half width $\approx 4000 \mathrm{~cm}^{-1}$ ). This difference in width suggests that the molecules of ThT bound to the amyloid fibrils are restricted to some subset of conformations with very uniform fluorescence properties. The fibrillar structure may favor particular conformations, probably those close to planarity. By contrast, the high viscosity of glycerol or the binding to BSA do not have such a selective effect. These spectral differences between ThT bound to A $\beta 42$ and ThT bound to BSA may be exploited for the discrimination of different types of peptide.

In aqueous solution and in chloroform there is an enhancement of the red emission resulting in band half widths that are nearly twice that in amyloid fibrils. This weak red emission becomes visible in the normalized spectra of these samples because of the much lower fluorescence quantum yield of the blue emission (Table 2). At $410 \mathrm{~nm}$ the perpendicular conformations are not effectively excited and in any case their emission is located at lower wavelengths (see Figure 1). Therefore this weak low-energy emission might be due to limiting ThT conformations or to an exiguous fluorescence of the TICT state. 


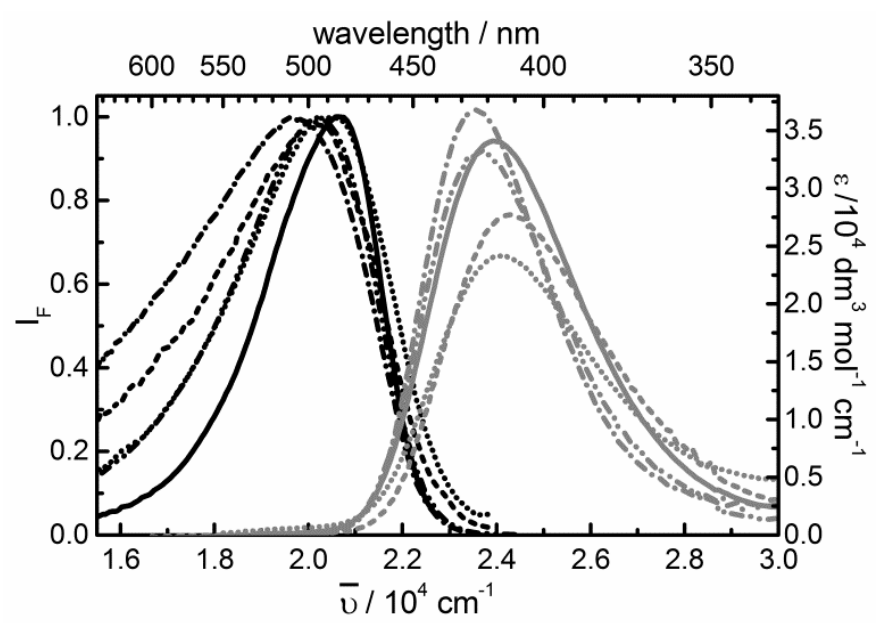

Figure 3. Absorption (grey lines) and normalized fluorescence emission spectra (black lines) of ThT in different media under excitation at $(410 \pm 10) \mathrm{nm}$. The spectra of ThT in PBS buffer (dashed), chloroform (dash-dotted) and 99\% glycerol (dash-dot-dotted) were measured directly. The spectra of ThT bound to BSA (dotted) and to A 342 (solid) are "pure” spectra obtained by global analysis of the corresponding series of absorption and emission spectra. (The spectra have been transformed to wavenumber taking into account the variation in bandpass $I_{F}(\bar{v})=\lambda^{2} I(\lambda)$ [34]. Spectra in the wavelength scale are given in Figure 3S in the SI.)

Using the absorption and emission "pure” spectra determined above for ThT bound to BSA and A $\beta 42$ and the experimental spectra of ThT in the different solvents and mixtures, the fluorescence quantum yields of ThT in the different media were calculated (Table 2 and open circles in Figure 4). The values obtained for the pure solvents agree quite well with reported data [12]. For glycerol-water mixtures we obtain significantly higher absolute quantum yields than those found in the literature [13]. This can be due to the different experimental conditions and quantum-yield standard used. Nevertheless, the viscosity dependence coincides with that reported, with an increase in $\phi$ of nearly 3 orders of magnitude from aqueous solution to $99 \%$ glycerol that is explained by the increasing hindrance of TICT formation as the solvent becomes more viscous (Figure 4 and Scheme 2). 
ThT bound to $A \beta 42$ shows the highest fluorescence quantum yield of all media (Table 2), with a value similar to those reported for other amyloid-type peptides [17,46,47]. In this case TICT formation is hindered due to the binding of the dye to the amyloid fibrils. When ThT binds to BSA the increase of $\phi$ is smaller. This might be due to a weaker dye-protein interaction or to the different binding mode of ThT with non fibrillar proteins.

The fluorescence lifetimes determined for ThT in the different solvents and mixtures are shown in Table 2 and Figure 4. In all cases two lifetimes were necessary to fit the experimental decays. In aqueous solution the main contribution is from a species with very short lifetime that is attributed to planar conformations undergoing a rapid conversion to the dark TICT state, whereas the second longer lifetime could be due to a small fraction of the brighter perpendicular conformations (Scheme 2). Similar lifetimes are obtained in chloroform, although in this case they have equal contributions to the total fluorescence, in contrast to aqueous solution.

For the glycerol-water mixtures also two lifetimes are obtained (Figure 4 and Table 2). The shorter one increases as the glycerol percentage is increased and contributes with about $90 \%$ to the measured fluorescence except at the highest glycerol percentages. The viscosity dependence of the lifetime is similar to that of the corresponding fluorescence quantum yield and can be explained likewise (Scheme 2). At low viscosities ThT has a short lifetime due to a conversion to the TICT state that is faster than the radiative deactivation. The twisting process is progressively more hindered as the viscosity is increased so that the TICT formation becomes slower and competes with the radiative deactivation leading to a longer lifetime. Neglecting the contribution of the second longer lifetime to the observed fluorescence, the nonradiative rate constant $k_{n r}=(1-\phi) / \tau_{1}$ can be estimated from $\phi$ and $\tau_{1}$ in these mixtures (black triangles in Figure 4). The results show that $k_{\mathrm{nn}}$, which is a measure of the conversion rate to the TICT state $k_{\mathrm{nr}, \mathrm{TICT}}$, drops very rapidly as the viscosity increases, reflecting the raising hindrance for the excited-state twisting process. This estimation is not valid for the highest volume percentages of glycerol since 
there the contribution of the second lifetime is not negligible. Similarly to the other solvents, this longer lifetime can be attributed to perpendicular conformations with relatively high fluorescence intensity. The lifetimes obtained for ThT in 99\% glycerol (Table 2) are compatible with those reported at other temperatures [13].

For BSA and A $\beta 42$ time-resolved fluorescence titrations were performed by varying the protein concentration at a constant dye concentration. All decays are biexponential (apart from minor contributions associated to BSA impurities) and show the same two lifetimes but increasing amplitudes as the concentration of the peptide is increased. These results indicate that there are two emitting species in the presence of $\mathrm{A} \beta 42$ (or BSA) that behave as expected for bound ThT since their concentrations increase as the peptide concentration is increased. These species must be assigned to two distinct binding sites of ThT in the peptides that are related to conformations with different fluorescence properties and probably different binding affinities. Further analysis of the amplitudes will allow us to extract information about these two binding sites. Best estimates of the lifetimes ( $\tau_{1}$ and $\tau_{2}$ ) and the amplitudes (A1 and A2) as obtained from global analysis of the series of fluorescence decays are given in Table 2 and Figure $4 \mathrm{~S}$ in the SI.

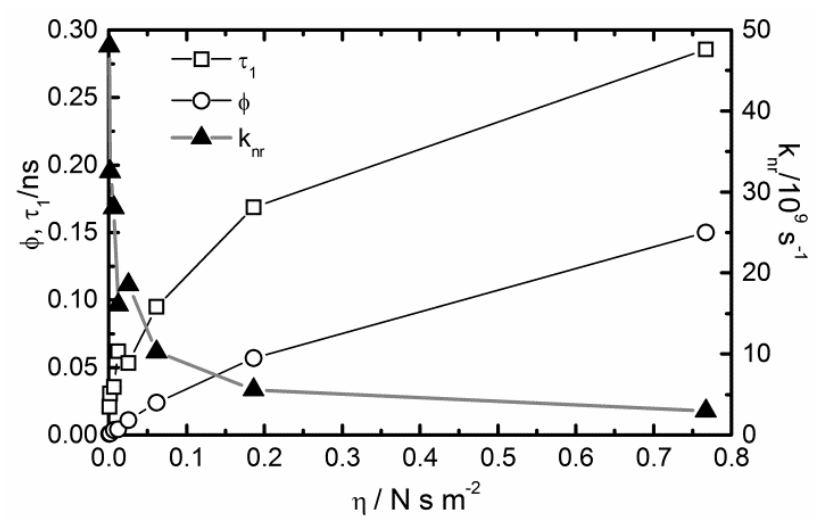

Figure 4. Fluorescence quantum yield $\phi$ (open circles), fluorescence lifetime $\tau_{1}$ (open squares) and nonradiative deactivation rate constant $k_{\mathrm{nr}}$ (black triangles) of ThT in glycerol-water mixtures versus viscosity. The volume percentages of glycerol are the same as in Figure 2 (9.9, 24.7, 49.5, 
59.4, 69.3, 79.2, 89.1 and 99.0\%) and correspond to increasing viscosity values. The fluorescence quantum yields were obtained from the spectra shown in Figures 2 and 2S. Fits of the decays yield a second longer lifetime with low contribution and great uncertainty ( $\tau_{2}=0.6 \pm 0.1 \mathrm{~ns}$ ) and two residual lifetimes associated to glycerol impurities. The nonradiative deactivation rate constant $k_{\mathrm{nr}}$ was calculated from $\phi$ and $\tau_{1}$.

Assuming independent complexation processes for the two binding sites, the macroscopic binding constant $K$ determined from the fluorescence intensity (Table 2 ) is the sum of the microscopic binding constants $K_{1}$ and $K_{2}$ corresponding to site 1 (associated with $\tau_{1}$ and A1) and site 2 ( $\tau_{2}$ and A2), respectively. Each amplitude is proportional to the fluorescence quantum yield and to the concentration of ThT at the corresponding binding site. Therefore, the fit of A1 and A2 to 1:1 complexation models (eq. (1)) with $K_{1}$ and $K_{2}$ as binding constants and under the constraint that $K=K_{1}+K_{2}$ (curves in Figure 4S) yields rough estimates of the microscopic binding constants and of the individual fluorescence intensities of ThT at the two binding sites (fluorescence percentages in Table 2).

For the binding of ThT with $\mathrm{A} \beta 42$ the value of $K_{1}$ is about 4 times higher than that of $K_{2}$, indicating that ThT binds preferably to site 1 . Nevertheless, when all the ThT is bound to A $\beta 42$ then $90 \%$ of the observed fluorescence is due to ThT molecules in site 2 (see percentages in Table 2), which also have the longer lifetime. According to the photophysical model proposed (Scheme 2), ThT bound to site 2 must have essentially a planar conformation with a strong steric hindrance for the excited-state twisting so that it emits efficiently from the locally excited state. Thus, ThT bound to site 1 may correspond to nearly perpendicular conformations, characterized by a shorter lifetime and lower fluorescence intensity. The fact that most of the fluorescence comes from those ThT molecules bound to site 2 explains the narrow fluorescence band of ThT bound to A $\beta 42$ (Figures 3 and $3 \mathrm{~S}$ in SI). 
According to these results, only one of five ThT molecules bound to $A \beta 42$ have the highly fluorescent conformation of site 2. This dominance of the emission of the weakly bound site is an inconvenience of this marker for the quantitative determination of amyloid fibrils, since the detected species has effectively a weak interaction with the fibrillar structure. It should be further investigated whether this effect is common to all amyloid fibrils. A recent study has found two binding sites of ThT with insulin and lysozyme fibrils but the second, weak-fluorescent site has much lower fluorescent quantum yield [47].

The behavior of ThT bound to BSA is quite different. $K_{1}$ is now approximately half of $K_{2}$, so that site 2 is favored. Lifetimes $\tau_{1}$ and $\tau_{2}$ have similar values as for ThT bound to A $\beta 42$ and can therefore be assigned to the same conformations. Nevertheless, the two binding sites have comparable contributions to the observed fluorescence (percentages in Table 2). This must be the reason for the broadening of ThT emission spectrum when it is bound to BSA in comparison to amyloid fibrils. Also the lower fluorescence quantum yield must be due to the significant contribution of the less fluorescent site 1 .

Steady-state anisotropy was determined for some high volume percentages of glycerol and for different concentrations of BSA and A $\beta 42$ (grey squares in Figure 2). Despite the relatively high deviations it can be observed that ThT anisotropy has similar values in all three media and they are close to the anisotropy limit of 0.40 . Moreover, these high anisotropy values are already reached at small concentrations of BSA or A $\beta 42$, since the observed fluorescence is only due to bound ThT as the free dye has very low quantum yield (see Table 2). Thus, the mean anisotropy is mostly independent of the fraction of bound dye which is again a disadvantage of ThT as a probe for the quantitative determinations of amyloid fibrils.

\section{Conclusions}


The detailed photophysical analysis confirms that the remarkable properties of ThT as fluorescence marker of amyloid fibrils are based on its behavior as molecular rotor. Nevertheless, the existence of a wide solvent-dependent distribution of ground-state conformations with very different photophysical behaviors makes ThT a highly complex probe with strong wavelength dependencies of all its fluorescence properties. The binding constant of ThT to A $\beta 42$ is rather low and is comparable to that with BSA. In both proteins ThT is bound to two distinct sites associated to different ThT conformations.

\section{Acknowledgements}

S.F. and M. H. A. thank the Xunta de Galicia and the Ministerio de Educación, Cultura e Deporte (Program "Ciencia sin Fronteras") for scholarship. M.N. and W.A. thank the Ministerio de Ciencia e Innovación and the Xunta de Galicia for their financial support (CTQ2010-21369, CN2012/314, GPC2013/052).

\section{References}

[1] Melanie R N. Techniques to study amyloid fibril formation in vitro. Methods 2004 9; 34(1): 151-60.

[2] Groenning M. Binding mode of Thioflavin $\mathrm{T}$ and other molecular probes in the context of amyloid fibrils_-current status. J. Chem. Biol. 2010; 3(1): 1-18.

[3] Amdursky N, Erez Y, Huppert D. Molecular rotors: what lies behind the high sensitivity of the thioflavin-T fluorescent marker. Acc. Chem. Res. 2012; 45(9): 1548-57.

[4] Ban T, Hamada D, Hasegawa K, Naiki H, Goto Y. Direct observation of amyloid fibril growth monitored by thioflavin T fluorescence. J. Biol. Chem. 2003; 278(19): 16462-5.

[5] Sabaté R, Saupe SJ. Thioflavin T fluorescence anisotropy: an alternative technique for the study of amyloid aggregation. Biochem. Biophys. Res.Commun. 2007;360(1):135-8.

[6] D’Amico M, Di Carlo MG, Groenning M, Militello V, Vetri V, Leone M. Thioflavin T promotes A $\beta$ (1-40) amyloid fibrils formation. J. Phys. Chem. Lett. 2012; 3(12): 1596-601.

[7] Reinke AA, Abulwerdi GA, Gestwicki JE. Quantifying prefibrillar amyloids in vitro by using a “Thioflavin-like” spectroscopic method. ChemBioChem 2010; 11(13): 1889-95.

[8] Selkoe DJ. Folding proteins in fatal ways. Nature 2003; 426(6968): 900-4. 
[9] Gong YS, Chang L, Viola KL, Lacor PN, Lambert MP, Finch CE, et al. Alzheimer's diseaseaffected brain: presence of oligomeric $A \beta$ ligands (ADDLs) suggests a molecular basis for reversible memory loss. Proc. Natl. Acad. Sci. U.S.A. 2003; 100(18): 10417-22.

[10] Haass C, Selkoe DJ. Soluble protein oligomers in neurodegeneration: lessons from the Alzheimer's amyloid [beta]-peptide. Nat. Rev. Mol. Cell Biol. 2007; 8(2): 101-12.

[11] Srivastava A, Singh PK, Kumbhakar M, Mukherjee T, Chattopadyay S, Pal H, et al. Identifying the bond responsible for the fluorescence modulation in an amyloid fibril sensor. Chem. Eur. J. 2010; 16(30): 9257-63.

[12] Maskevich AA, Stsiapura VI, Kuzmitsky VA, Kuznetsova IM, Povarova OI, Uversky VN, et al. Spectral properties of Thioflavin $\mathrm{T}$ in solvents with different dielectric properties and in a fibril-incorporated form. J. Proteome Res. 2007; 6(4): 1392-401.

[13] Stsiapura VI, Maskevich AA, Kuzmitsky VA, Uversky VN, Kuznetsova IM, Turoverov KK. Thioflavin $\mathrm{T}$ as a molecular rotor: fluorescent properties of Thioflavin $\mathrm{T}$ in solvents with different viscosity. J. Phys. Chem. B 2008; 112(49): 15893-902.

[14] Stsiapura VI, Maskevich AA, Tikhomirov SA, Buganov OV. Charge Transfer process determines ultrafast excited state deactivation of Thioflavin T in low-viscosity solvents. J. Phys. Chem. A 2010; 114(32): 900-4.

[15] Naik L, Naik AB, Pal H. Steady-state and time-resolved emission studies of Thioflavin-T. J. Photochem. Photobiol. A 2009; 204(2): 161-7.

[16] Sen P, Fatima S, Ahmad B, Khan RH. Interactions of Thioflavin T with serum albumins: spectroscopic analyses. Spectroc. Acta Pt A: Molec. Biomolec. Spectr. 2009; 74(1): 94-9.

[17] Sulatskaya AI, Maskevich AA, Kuznetsova IM, Uversky VN, Turoverov KK. Fluorescence quantum yield of Thioflavin $\mathrm{T}$ in rigid isotropic solution and incorporated into the amyloid fibrils. PLoS One 2010; 5(10): e15385.

[18] Dutta Choudhury S, Mohanty J, Upadhyaya HP, Bhasikuttan AC, Pal H. Photophysical studies on the noncovalent interaction of Thioflavin $\mathrm{T}$ with cucurbit [n] uril macrocycles. J. Phys. Chem. B 2009; 113(7): 1891-8.

[19] Choudhury SD, Mohanty J, Pal H, Bhasikuttan AC. Cooperative metal ion binding to a cucurbit [7] uril- Thioflavin T complex: Demonstration of a stimulus-responsive fluorescent supramolecular capsule. J. Am. Chem. Soc. 2010; 132(4): 1395-401.

[20] Singh PK, Kumbhakar M, Pal H, Nath S. Viscosity effect on the ultrafast bond twisting dynamics in an amyloid fibril sensor: Thioflavin-T. J. Phys. Chem. B 2010; 114(17): 5920-7.

[21] Sabate R, Rodríguez-Santiago L, Sodupe M, Saupe SJ, Ventura S. Thioflavin-T excimer formation upon interaction with amyloid fibers. Chem. Commun. 2013; 49: 5745-7.

[22] Khurana R, Coleman C, Ionescu-Zanetti C, Carter SA, Krishna V, Grover RK, et al. Mechanism of Thioflavin T binding to amyloid fibrils. J. Struct. Biol. 2005; 151(3): 229-38.

[23] Groenning M, Norrman M, Flink JM, van de Weert M, Bukrinsky JT, Schluckebier G, et al. Binding mode of Thioflavin T in insulin amyloid fibrils. J. Struct. Biol. 2007; 159(3): 483-97. 
[24] Krebs MRH, Bromley EHC, Donald AM. The binding of thioflavin-T to amyloid fibrils: localisation and implications. J. Struct. Biol. 2005 1; 149(1): 30-7.

[25] Rodríguez-Rodríguez C, Rimola A, Rodríguez-Santiago L, Ugliengo P, Álvarez-Larena Á, Gutiérrez-de-Terán H, et al. Crystal structure of Thioflavin-T and its binding to amyloid fibrils: insights at the molecular level. Chem. Commun. 2010; 46(7): 1156-8.

[26] Wolfe LS, Calabrese MF, Nath A, Blaho DV, Miranker AD, Xiong Y. Protein-induced photophysical changes to the amyloid indicator dye Thioflavin T. Proc. Natl. Acad. Sci. U.S.A. 2010; 107(39): 16863.

[27] Duboisset J, Ferrand P, He W, Wang X, Rigneault H, Brasselet S. Thioflavine-T and Congo Red reveal the polymorphism of insulin amyloid fibrils when probed by polarization-resolved fluorescence microscopy. J. Phys. Chem. B 2013; 117(3): 784-8.

[28] LaFerla FM, Green KN, Oddo S. Intracellular amyloid-[beta] in Alzheimer's disease. Nat. Rev. Neurosci. 2007; 8(7): 499-509.

[29] Chen Y, Glabe CG. Distinct early folding and aggregation properties of Alzheimer amyloid$\beta$ peptides $A \beta 40$ and $A \beta 42$ : stable trimer or tetramer formation by $A \beta 42$. J. Biol. Chem. 2006; 281(34): 24414-22.

[30] Walsh DM, Selkoe DJ. A $\beta$ Oligomers - a decade of discovery. J. Neurochem. 2007; 101(5): 1172-84.

[31] Harper JD, Lansbury Jr PT. Models of amyloid seeding in Alzheimer's disease and scrapie: mechanistic truths and physiological consequences of the time-dependent solubility of amyloid proteins. Annu. Rev. Biochem. 1997; 66(1): 385-407.

[32] Jarrett JT, Berger EP, Lansbury PT. The carboxy terminus of the $\beta$ amyloid protein is critical for the seeding of amyloid formation: Implications for the pathogenesis of Alzheimer's disease. Biochemistry 1993; 32(18): 4693-7.

[33] Stine WB,Jr, Dahlgren KN, Krafft GA, LaDu MJ. In vitro characterization of conditions for amyloid-beta peptide oligomerization and fibrillogenesis. J. Biol. Chem. 2003; 278(13): 1161222.

[34] Lakowicz JR. Principles of fluorescence spectroscopy. USA: Springer; 2006.

[35] Bordello J, Novo M, Al-Soufi W. Exchange-dynamics of a neutral hydrophobic dye in micellar solutions studied by Fluorescence Correlation Spectroscopy. J. Colloid Interface Sci. 2010; 345: 369-76.

[36] Al-Soufi W, Novo M, Mosquera M. Principal component global analysis of fluorescence and absorption spectra of 2-(2'-hydroxyphenyl)benzimidazole. Appl. Spectrosc. 2001; 55(5):630-6.

[37] Weast RC. CRC handbook of chemistry and physics. Boca Raton, Florida: CRC Press; 1986.

[38] Cheng N. Formula for the viscosity of a glycerol-water mixture. Ind .Eng. Chem. Res. 2008; 47(9): 3285-8. 
[39] The Dow Chemical Company. Refractive index of glycerine-water solutions. Available at: http://www.dow.com/optim/optim-advantage/physical-properties/refractive.htm. Accessed March 15, 2014.

[40] Voropai E, Samtsov M, Kaplevskii K, Maskevich A, Stepuro V, Povarova O, et al. Spectral properties of Thioflavin $\mathrm{T}$ and its complexes with amyloid fibrils. J. Appl. Spectrosc. 2003; 70(6): 868-74.

[41] Stsiapura VI, Maskevich AA, Kuzmitsky VA, Turoverov KK, Kuznetsova IM. Computational study of Thioflavin T torsional relaxation in the excited state. J. Phys. Chem. A 2007; 111(22): 4829-35.

[42] Dey JK, Dogra SK. Solvatochromism and prototropism in 2-(aminophenyl) benzothiazoles. Bull. Chem. Soc. Jpn. 1991; 64(10): 3142-52.

[43] Rios Vazquez S, Rios Rodriguez MC, Mosquera M, Rodriguez-Prieto F. Excited-state intramolecular proton transfer in 2-(3'-Hydroxy-2'-pyridyl)benzoxazole. Evidence of coupled proton and charge transfer in the excited state of some o-Hydroxyarylbenzazoles. J. Phys. Chem. B 2007; 111(10): 1814-26.

[44] Demchenko AP, Tang K, Chou P. Excited-state proton coupled charge transfer modulated by molecular structure and media polarization. Chem. Soc. Rev. 2013; 42(3): 1379-408.

[45] Eisert R, Felau L, Brown LR. Methods for enhancing the accuracy and reproducibility of Congo Red and Thioflavin T assays. Anal.Biochem. 2006;353:144-6.

[46] Sulatskaya AI, Kuznetsova IM, Turoverov KK. Interaction of Thioflavin T with amyloid fibrils: stoichiometry and affinity of dye binding, absorption spectra of bound dye. J. Phys. Chem. B 2011; 115(39): 11519-24.

[47] Kuznetsova IM, Sulatskaya AI, Uversky VN, Turoverov KK. Analyzing Thioflavin T binding to amyloid fibrils by an equilibrium microdialysis-based technique. PloS one 2012; 7(2): e30724. 